\title{
Doença renal crônica e depressão: um estudo psicossociológico com pacientes em hemodiálise ${ }^{1}$
}

\author{
Chronic kidney disease and depression: a psychosociological study with hemodialysis \\ patients
}

\author{
Fabrycianne Gonçalves Costa \\ Maria da Penha de Lima Coutinho 3
}

\begin{abstract}
RESUMO: Este estudo objetivou apreender as representações sociais acerca da Doença Renal Crônica (DRC) e da depressão, elaboradas por pacientes sob tratamento de hemodiálise com e sem sintomas depressivos. Participaram deste estudo 50 pacientes, de ambos os sexos, com idade média de 46,05 anos e DP=13,4, que responderam a um questionário sociodemográfico, a Escala Hospitalar de Ansiedade e Depressão e a Técnica de Associação Livre de Palavras. Os dados foram analisados por meio da estatística descritiva e análise fatorial de correspondência. Os resultados apontaram que $20 \%$ dos pacientes apresentaram sintomas depressivos. A DRC foi representada como sinônimo de rim paralisado, associada às dificuldades do tratamento e considerada "um pesadelo". Os pacientes também expressaram a falta de conhecimento acerca das causas que desencadeiam a doença renal. A depressão foi objetivada nos sintomas: falta de vontade, choro e agonia.
\end{abstract}

Palavras-chave: representação social; depressão; doença renal crônica; hemodiálise; testes de associação de palavras.

ABSTRACT: This study aimed to apprehend the social representations of Chronic Renal Failure (CRF) and depression, elaborated by patients on hemodialysis with and without symptoms of depression. Fifteen patients on hemodialysis participated in this study, of both sexes, with a mean age of 46.05 years, SD $=13.4$, who answered to a sociodemographic questionnaire, the Hospital Anxiety and Depression Scale and the Free Word Association Test. The data were analyzed using descriptive statistics and factorial correspondence analysis. The results indicated that $20 \%$ of patients had depressive symptoms. The CRF was represented as synonymous of paralyzed kidney, associated with the treatment difficulties and its which was considered "a nightmare". Patients also expressed on the ignorance of the causes which lead to kidney disease. The depression has been objectified in the symptoms: lack of desire, crying and agony.

Keywords: social representation; depression; chronic kidney disease; hemodialysis; word association tests.

\section{Introdução}

A partir das últimas três décadas do século XX, observou-se um crescente interesse no entendimento acerca do binômio saúde-doença, considerando-se os fatores biopsicossociais que decorrem desse fenômeno. Neste direcionamento, a literatura vem enfatizando a associação entre distúrbios psicoafetivos e doenças crônicas, a exemplo da depressão e da Doença Renal Crônica (DRC), que passou a ser estudada, aproveitando-se as contribuições da Psicologia da saúde e da Psicologia social (Nifa \& Rudnicki, 2010; Kimmel, Cohen \& Peterson,

\footnotetext{
${ }^{1}$ Agencia de fomento: Capes

2 Doutoranda em Psicologia Social pela Universidade Federal da Paraíba (UFPB) - João Pessoa, PB, Brasil. E-mail: fabrycianne@gmail.com.

3 Doutora em Psicologia Clínica pela Universidade de São Paulo; Professora da Pós-Graduação em Psicologia Social da Universidade Federal da Paraíba (UFPB) - João Pessoa, PB, Brasil.
} 
2008).

A DRC é caracterizada pela perda progressiva, irreversível e multifatorial da função renal que pode desencadear alterações nos diversos sistemas do organismo, contribuindo para o fracasso da capacidade do corpo em manter os equilíbrios metabólicos e hidroeletrolíticos (Kidney Disease Improving Global Outcomes- KDIGO, 2013). Na medida em que a função renal diminui, os produtos finais do metabolismo proteico, que são excretados pela urina, passam a acumular-se no sangue. Por ser uma doença lenta e progressiva, a perda do funcionamento do rim resulta em processos adaptativos que, até certo ponto, mantêm as pessoas sem sintomas. Frequentemente, esses sintomas só surgem quando cerca de $50 \%$ da função renal já foi perdida. Nesse estágio da doença, os rins apresentam apenas 10 a $12 \%$ de sua capacidade funcional, sendo necessário tratamento com urgência (Ribeiro et al., 2008).

Nesse caso, rotineiramente a hemodiálise é o tratamento adotado. Trata-se de um processo mecânico e extracorpóreo que consiste na remoção de substâncias tóxicas e excesso de líquido do organismo. Em geral, esse procedimento é realizado em hospitais, três vezes por semana e cada sessão demora entre três e quatro horas. Além desse tratamento, os pacientes devem ingerir medicamentos e seguir dietas, restringindo especialmente a quantidade de líquido (Rudnicki, 2014).

O indivíduo acometido da doença renal é conduzido a conviver diariamente com uma doença incurável, que o leva a uma forma de tratamento dolorosa, de longa duração e que provoca, juntamente com a evolução da doença e suas complicações, ainda maiores limitações e alterações de grande impacto, que repercutem tanto na sua própria vida quanto na do grupo familiar, afetando as várias dimensões do ser humano sejam elas de ordem física, psicológica, econômica ou social (Ramos, Queiroz, \& Jorge, 2008).

Sendo assim, a constante exposição a fatores adversos inerentes à terapêutica da síndrome renal, como o tempo gasto nas sessões de hemodiálise, constantes consultas médicas, exames laboratoriais, dietas e expectativa de transplante, associados à frequente permanência em ambientes hospitalares, têm contribuído para o surgimento de comorbidades, como é o caso da sintomatologia depressiva (Coutinho \& Costa, 2015; Ferreira \& Anes, 2010).

A literatura aponta que a depressão parece ser a complicação psíquica mais frequente nos pacientes em tratamento de hemodiálise (Nifa \& Rudnicki, 2010; Kimmel et al., 2008). Conforme Smith, Hong e Robson (1985), a prevalência da sintomatologia depressiva nessa população pode atingir um índice de até $100 \%$, embora estudos mais recentes tenham apontado prevalências menos abarcantes, a exemplo de Garcia, Veiga e Motta (2010) e Costa, Coutinho, Melo e Oliveira (2014), que indicam uma oscilação entre 56,3\% a 68\%. Outros estudos assinalam uma variação ainda menor entre $10 \%$ e $30 \%$ (Andrade, Sesso \& Diniz, 2015; Kimmel et al., 2008).

A depressão é considerada uma patologia classificada dentro dos transtornos de humor, sendo esta reconhecida como um problema prioritário na saúde pública. Conforme os dados da Organização Mundial de Saúde - OMS (2016) estima-se que mais de 350 milhões de pessoas, em todo o mundo, sofram de depressão. De acordo com Kaplan, Sadock e Grebb (2007) o transtorno depressivo é caracterizado por alterações afetivas, cognitivas, volitivas e psicomotoras, comprometendo, assim, o funcionamento global do indivíduo, interferindo em seu cotidiano e nas suas relações sociais. 
Neste estudo, a depressão é vista como um sofrimento psíquico desencadeado por um acontecimento desagradável (acometimento da DRC) que interfere significativamente na diminuição da qualidade de vida, na produtividade e capacitação social do indivíduo, compreendida como depressão reativa segundo o Manual Diagnóstico e Estatístico de Transtornos Mentais- DSM- V (APA, 2014). No senso comum, o termo depressão indica desde simples alterações psicológicas ou perturbações psiquiátricas graves até flutuações de humor ou de caráter, podendo nomear tanto um estado afetivo normal quanto um sintoma, uma síndrome ou várias doenças (Ribeiro, Coutinho \& Nascimento, 2010).

Nesse sentido, o impacto do diagnóstico e o tratamento da hemodiálise podem levar o indivíduo a redimensionar tudo o que foi vivido anteriormente, em um processo de revisão de si e de suas relações, assim ocorrendo um processo de busca pela adaptação biopsicossocial, utilizando o saber comum para criação de teorias, procurando respostas para os acontecimentos cotidianos, passando por etapas de elaboração e reformulação contínua transladada pelos próprios sujeitos que experienciam a realidade (Santos, Barbosa \& Alves Júnior, 2005).

São diversos os significados que passam no imaginário das pessoas afetadas pela doença renal, indo desde o impacto do diagnóstico, associado ao reconhecimento da gravidade da doença e do tratamento até suas consequências, como os efeitos medicamentosos e os limites nos hábitos alimentares e na vida social. Em geral, essas situações adversas provocam medo, dúvidas e insegurança quanto à cura e à possibilidade de viver (Ramos et al., 2008).

Nesse sentido, o modo como os pacientes representam a doença renal e a depressão pode direcionar seus comportamentos, comunicações e práticas cotidianas, nos auxiliando a entender como esses indivíduos interpretam a nova realidade de suas vidas permeada pela doença renal. Em face dessas premissas, esta pesquisa objetivou apreender as representações sociais acerca da Doença Renal Crônica e da depressão, elaboradas por pacientes sob tratamento de hemodiálise com e sem sintomas depressivos, tendo como eixo norteador a abordagem psicossocial da Teoria das Representações Sociais (TRS).

Segundo Moscovici (2012), as representações sociais (RS) são conjuntos simbólicos/práticos/dinâmicos, cujo status é o de uma produção e não de uma reprodução. Isso significa dizer que constituem não uma simples reação a estímulos exteriores, mas sim a escolha e a utilização, a partir de repertório circulante na sociedade, de informações destinadas à interpretação e à elaboração do real. Assim, representar um objeto, pessoa ou coisa não consiste apenas em desdobrá-los, repeti-los ou reproduzi-los, mas em reconstruílos, retocá-los e modificá-los.

Nesse direcionamento, ter acesso às representações sociais de um objeto social é tentar compreender as formas que os indivíduos utilizam para criar, transformar e interpretar uma problemática vinculada à sua realidade, bem como conhecer seus pensamentos, sentimentos, percepções e experiências de vida compartilhada, de acordo com a classe social a que pertencem e às instituições às quais estão vinculados (Coutinho, 2005).

Na perspectiva psicossocial, observam-se estudos que abordaram diferentes construtos para identificar as RS no contexto da doença renal, por exemplo, sobre as RS acerca do cuidado, estudadas por Ramos et al. (2008), assim como acerca do adoecimento (Nascimento, Campos, Mantovani \& Cassi, 2015). Além disso, Coutinho e Costa (2015) vislumbraram as RS da depressão no contexto de familiares e pacientes renais. O tratamento, 
especificamente a hemodiálise, também foi objeto de investigação no âmbito psicossociológico (Campos \& Turato, 2010; Costa, Silva, Alves, Silva \& Leite, 2007). Demais autores estudaram acerca das RS da DRC e do transplante (Palmeira, 2009; Quintana \& Muller, 2006).

Não obstante a existência dos estudos supracitados, percebe-se pouca evidência do construto da depressão sob o enfoque psicossociológico no contexto da DRC. Em geral, observa-se que os pesquisadores têm privilegiado quantificar a depressão nas pessoas em tratamento de hemodiálise. Nesse direcionamento, optou-se por também se enfatizar a compreensão e explicação desse sofrimento psicoafetivo contemplando as RS.

Diante do exposto, a presente pesquisa possui grande relevância científica, sobretudo no que se refere à construção do conhecimento socialmente compartilhado pelos pacientes em hemodiálise acerca da DRC e da depressão. Esse material visa também fornecer subsídios aos profissionais da saúde contribuindo para elaboração de estratégias de prevenção e tratamento da doença renal que levem em consideração tanto os aspectos psicossociais quanto os físicos.

\section{Método}

\section{Tipo de estudo}

Tratou-se de um estudo exploratório e descritivo, de cunho qualiquantitativo.

\section{Local e participantes}

A pesquisa foi desenvolvida em três instituições hospitalares que disponibilizam o tratamento da hemodiálise para adultos e mantêm convênio com o Sistema Único de Saúde (SUS), localizadas na cidade de João Pessoa/PB.

Participaram deste estudo 50 pacientes com DRC, escolhidos de forma não probabilística e por conveniência, com idades entre 20 e 73 anos ( $M=46,05$; $D P=13,4)$, sendo a maioria do sexo feminino (52\%) e $33 \%$ casados. Utilizou-se como critérios de inclusão: estar em tratamento de hemodiálise e ter idade mínima de 18 anos.

\section{Instrumentos}

Para a obtenção dos dados utilizou-se um questionário sociodemográfico, a Técnica de Associação Livre de Palavras (TALP) e a Escala Hospitalar de Ansiedade e Depressão (HADS). O primeiro instrumento foi utilizado com a finalidade de obter o perfil característico da amostra, assim como estabelecer parte das variáveis fixas (idade, sexo e estado civil) que compuseram o banco de dados processado pelo programa computacional Tri-Deux-Mots.

Fez-se uso da TALP, tendo como estímulos indutores: "doença renal crônica", "depressão" e "eu mesmo". A escolha desse último termo indutor teve o intuito de saber qual é o campo semântico que os participantes constroem enquanto autorreferência, possibilitando visualizar se os mesmos se veem como depressivos ou não.

Esse instrumento permite a atualização de elementos implícitos ou latentes que seriam perdidos ou mascarados nas produções discursivas. Trata-se de uma técnica projetiva 
amplamente utilizada no âmbito da Psicologia Social, principalmente quando se trabalha com o aporte teórico das RS, a qual permite evidenciar universos semânticos que refletem os universos comuns de palavras em face de diferentes estímulos e sujeitos ou grupos (Nóbrega \& Coutinho, 2011).

Utilizou-se ainda a HADS, instrumento adaptado à população brasileira por Botega, Bio, Zomignani, Garcia e Pereira (1995). Essa escala possui 14 itens, sete voltados para a avaliação da ansiedade (HADS-A) e sete para a depressão (HADS-D), que apresentam os respectivos coeficientes de alfa de Cronbach, 0,68 e 0,77. Cada item pode ser pontuado de zero a três, compondo uma pontuação de $0 \pm 21$, sendo recomendável " 8 " como ponto de corte (Marcolino et al., 2007; Botega et al., 1995).

Em um estudo realizado por Marcolino et al. (2007) para avaliar a validade de critério e da confiabilidade com pacientes no pré-operatório, comparando com as escalas de Beck de ansiedade e depressão, constatou-se que a correlação da HADS variou de 0,6 a 0,7, considerada uma correlação de média a forte, apresentando índices de consistência interna recomendáveis para instrumentos de triagem. Neste estudo, utilizou-se a subescala que se refere à depressão (HADS-D), como instrumento de screening, de modo que o resultado dos participantes com e sem sintomatologia depressiva computaram como uma das variáveis fixas no software Tri-Deux-Mots.

\section{Procedimentos éticos e de coleta de dados}

Este estudo foi realizado considerando as condições éticas estabelecidas pela Comissão do Conselho Nacional de Saúde, pertinentes a pesquisas envolvendo seres humanos, de acordo com a Resolução n 466/2012 e com constituição designada pela Resolução n 246/97, sendo aprovado pelo Comitê de Ética em Pesquisa do Centro de Ciências da Saúde, da Universidade Federal da Paraíba, sob o protocolo de n³92/11.

Quanto ao procedimento de coleta de dados, os pacientes foram abordados tanto na sala de espera, quanto na máquina, quando estavam dialisando. Inicialmente, cada paciente assinou um Termo de Consentimento Livre e Esclarecido, e, na sequência, procedeu-se à administração dos instrumentos, obedecendo-se à seguinte ordem: primeiro, a TALP; em seguida o questionário sociodemográfico e a HADS-D. Os instrumentos foram aplicados individualmente e oralmente sendo lidos pelos pesquisadores, devido à incapacidade de alguns participantes em responder por escrito. O tempo médio de aplicação dos instrumentos foi de 20 minutos e a pesquisa ocorreu no período de dezembro de 2011 a março de 2012.

\section{Análise dos dados}

Os dados provenientes do questionário sociodemográfico e da HADS-D foram analisados por meio do Pacote Estatístico para as Ciências Sociais (SPSS - 19.0), sendo utilizada a estatística descritiva (média, desvio padrão e frequências) para descrever a amostra estudada e verificar a prevalência da depressão.

Os dados coletados a partir da TALP foram processados pelo software Tri-Deux-Mots, versão 2.2, seguindo as cinco etapas do programa, a saber: IMPMOT (gera o dicionário e a frequência absoluta de palavras), TABMOT (versa em fazer a tabulação dos dados), ECAPEM (realiza o cruzamento entre as variáveis fixas (idade, sexo, estado civil e presença ou ausência 
de sintomas depressivos) e de opinião (DRC, depressão e eu mesmo), resultando como cada modalidade irá colaborar na construção do fator, ANECAR (gera o arquivo com as informações da Análise Fatorial de Correspondência (AFC)) e PLANFA (responsável pela produção do gráfico com as coordenadas ou fatores). Na sequência, o material foi analisado por meio da AFC, na qual é possível destacar os eixos F1 e F2, que explicam as modalidades de respostas, revelando as estruturas constituídas de elementos do campo representacional advindos do cruzamento tanto dos estímulos indutores quanto das variáveis fixas (Nóbrega \& Coutinho, 2011).

\section{Resultados e discussão}

Os resultados advindos da HADS-D registraram que $20 \%$ dos participantes apresentaram sintomas de depressão, com uma pontuação que variou de 8 a 18 pontos, com média de 5,36 ( $D P=3,4)$. Esta prevalência é concordante com outros estudos realizados com doentes renais, cujas porcentagens se situam entre 10 e 30\% (Andrade et al., 2015; Kimmel et al., 2008).

Esses dados assinalam que os pacientes com DRC, sob o tratamento de hemodiálise, estão mais suscetíveis de serem atingidos pela sintomatologia depressiva, quando comparados à população em geral, pois a literatura preconiza que aproximadamente $5 \%$ das pessoas no mundo sofram de depressão (OMS, 2016). Quando se trata de pacientes com DRC, a sintomatologia depressiva parece estar relacionada a mudanças na qualidade de vida decorrentes da diminuição da imunidade e da capacidade funcional, relaxamento dos cuidados pessoais e na adesão aos tratamentos e dietas. Consequentemente, esses problemas acarretam o aumento do número de consultas ambulatoriais, internações e até mortalidade (Chilcot, Wellsted, Silva-Gane \& Farrington, 2008; Cukor, Coplan, Brown, Peterson \& Kimmel, 2008).

Diante do exposto, fazem-se necessários maiores cuidados na atenção, promoção e prevenção da DRC, com o intuito de melhorar os programas de políticas públicas voltados para a conscientização da comunidade, em geral, no que se refere aos aspectos que predispõem ao desencadeamento dessa doença, tais como: fatores genéticos, hipertensão arterial, diabetes, obesidade, proteinúria, tabagismo, entre outros, com o intuito de minimizar a morbimortalidade dessa população (Costa \& Coutinho, 2016).

Em relação às representações sociais advindas da TALP, apresentadas na Figura 1, propiciam uma leitura representacional das variações semânticas associadas aos estímulos indutores: DRC, depressão e eu mesmo, elaboradas por pacientes sob tratamento de hemodiálise com e sem sintomas depressivos revelando as aproximações e os distanciamentos das modalidades de construção dos eixos ou fatores (F1 e F2). O somatório desses dois fatores evidenciou um poder explicativo de $90,7 \%$ da variância total das respostas, sendo que o F1 apresentou 52,0\%, e o F2, 38,7\%, demonstrando, portanto, parâmetros estatísticos com consistência interna e fidedignidade, tendo em vista pesquisas realizadas no âmbito das RS (Nóbrega \& Coutinho, 2011).

Conforme os resultados da análise do Tri-Deux-Mots encontraram-se 504 palavras evocadas como respostas aos estímulos indutores, das quais 214 foram diferentes e, entre as palavras referidas, 17 integram o plano fatorial, de acordo com a distribuição relativa de cada uma para o espaço fatorial. Nesse direcionamento, salienta-se que a frequência mínima para cada palavra evocada referente aos estímulos indutores configurada no plano fatorial foi de 
quatro coocorrências. Os resultados coletados possibilitaram a apreensão de significados do conhecimento prático, por meio do cruzamento das variáveis fixas e de opiniões, conforme pode ser observado no plano fatorial.

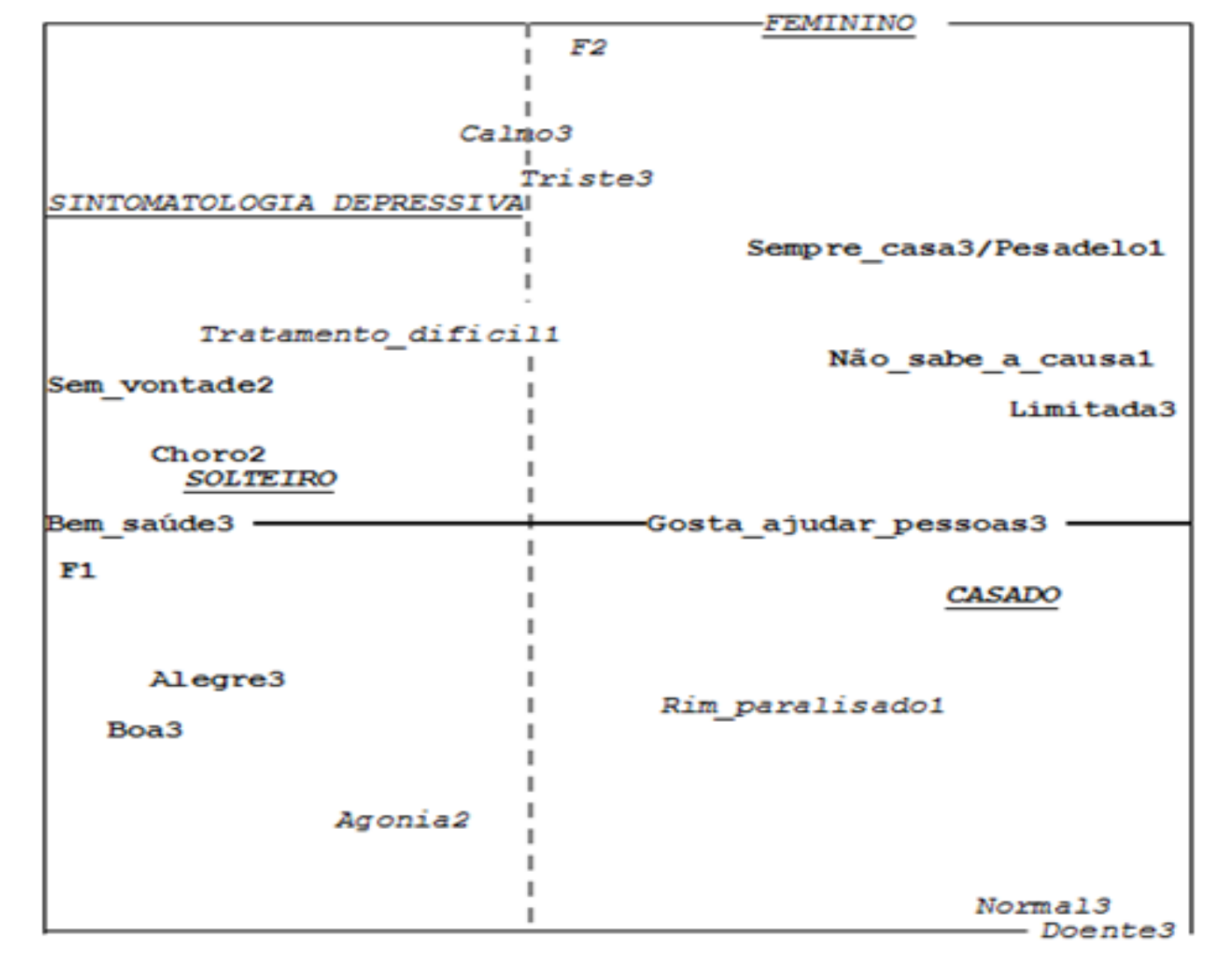

Figura 1 - Plano fatorial de correspondência das representações sociais acerca da DRC e da depressão, elaboradas por pacientes.

Legenda:

Fator 1(F1), em negrito, localiza-se no eixo horizontal à direita e à esquerda.

Fator 2(F2), em itálico, localiza-se no eixo vertical superior e inferior.

Estímulos indutores: 1= doença renal crônica; 2= depressão e 3= eu mesmo.

No fator 1 (F1), linha horizontal em negrito, do plano fatorial, observaram-se as construções em função do estado civil. Do lado esquerdo, situam-se as evocações dos participantes solteiros; em relação ao primeiro estímulo indutor "DRC", não foram observadas evocações no plano fatorial, possivelmente devido à pouca coocorrência do referido estímulo, conforme registro do subprograma ANECAR do Tri-Deux-Mots. No que se refere ao estímulo indutor "depressão", este foi objetivado nos elementos "sem vontade" e "choro" e ancorados nas categorias psicoafetiva e comportamental. Supõe-se que tais expressões manifestam as alterações fisiológicas decorrentes da depressão. Essas evidências estão em congruência com os achados encontrados por Saraiva e Coutinho (2008).

Quanto ao terceiro estímulo indutor "eu mesmo", os participantes solteiros se definiram como pessoas "saudáveis", "alegres" e "boas", compondo um campo semântico ancorado na esfera psicossocial. Tais elucidações remetem que apesar da doença esses pacientes se veem numa perspectiva positiva diante da vida. Os resultados supracitados estão em conformidade com os de Oliveira e Marques (2011) ao referirem que a maioria dos pacientes entrevistados em seu estudo se mostraram otimistas diante do enfrentamento da doença. 
Ainda no F1, do lado direito, posicionam-se as construções dos pacientes casados. Para estes, a DRC foi objetivada em "não sabem a causa" e "pesadelo" ancorados nas esferas físico-orgânico e emocional, respectivamente.

O desconhecimento sobre a causa da DRC se deve, muitas vezes, ao início insidioso e assintomático dessa doença, pois os seus sinais e sintomas aparecem silenciosamente, e, em alguns casos, quando a doença é detectada já se encontra crônica, sem chances de retorno da saúde. Conforme KDIGO (2013), devido à doença renal ser assintomática os indivíduos desconhecem sua existência até seu quadro clínico se apresentar bastante avançado, exigindo dos pacientes, com urgência, um tratamento para substituir a função renal. De acordo com a pesquisa de Campos e Turato (2010), os pacientes renais atribuíram à cultura vigente na sociedade a visão de desconhecimento acerca das causas da doença renal e, por conseguinte, a dificuldade de encarar com certa naturalidade o tratamento.

A doença renal foi ainda ancorada na dimensão emocional, objetivada como "pesadelo"; nesse caso, para essas pessoas, conviver com uma doença incurável e ter a necessidade de cumprir os tratamentos que a mesma exige; fuga da realidade, predominando o desejo de despertar desse sonho desagradável.

Santos e Valadares (2011) destacam que a forma como a pessoa recebe a notícia da doença e do tratamento pode gerar frustração, decepção e medo que pode gerar uma sensação de pesadelo, fazendo com que o paciente tente negar a realidade, da qual não pode se afastar, sob risco de morrer. $O$ choque maior é frente às primeiras informações, as quais soam como ataques, transformadas numa infindável dúvida pela ação do medo.

As representações sociais acerca da DRC elaboradas pelos participantes desta pesquisa estão de acordo com os dados constatados por Ramos et al. (2008), os quais evidenciaram que adolescentes em tratamento dialítico representaram a doença como sinônimo de diálise e preocupação, ressaltando que fazer hemodiálise representa uma forma de cuidado essencial à sua vida. Água, dieta, dedicação, proibições, responsabilidade e medicação também foram ancoradas no autocuidado, focalizando assim na tríade terapêutica: dieta, medicação e diálise.

Em relação ao segundo estímulo indutor "depressão", as evocações não foram suficientes para constar no plano fatorial, conforme o registro do subprograma PLANFA do Tri-Deux-Mots. Quanto ao último estímulo "eu mesmo", foi possível perceber que os pacientes casados se caracterizam como pessoas "limitadas" que estão "sempre em casa"; no entanto, "gostam de ajudar os outros". Essas elucidações se encontram ancoradas nas categorias comportamental e relações sociais. É possível inferir, que esses participantes fazem referência ao comportamento adotado frente às implicações do tratamento, nesse caso, por tratar-se da hemodiálise, que é um tratamento extenuante e requer tempo, o qual limita essas pessoas em suas atividades laborais, de lazer e cotidianas, contribuindo para que as mesmas fiquem mais tempo em suas residências.

Esses resultados se coadunam com os de Spinola e Gonçalves (2012) quando afirmam que o tratamento da DRC pode provocar restrições pela dependência do paciente em realizálo quatro horas por dia, três vezes por semana. Segundo estes autores as limitações mais frequentes são: mudanças no status social, laboral e financeiro; atividades sexuais. Outros fatores limitantes das atividades cotidianas são as dores, aplicar excesso de peso ou dormir sobre o braço da fistula arteriovenosa. O fato de preferirem ficar mais tempo em casa também pode estar relacionado à autoimagem prejudicada, devido às alterações provocadas 
no corpo tais como: coloração da pele, perda de peso, cicatrizes, entre outras.

O segundo fator (F2), eixo vertical do plano fatorial, reflete o pensamento coletivo dos participantes em função da sintomatologia da depressão e do sexo. Na parte superior do gráfico, situam-se as reproduções das mulheres com sintomas depressivos. Para essas participantes o estímulo indutor "DRC" foi objetivado enquanto doença de "difícil tratamento", e ancorada na esfera biopsicossocial, o que significa que o tratamento não depende apenas do seu aspecto medicamentoso, incluindo um conjunto de medidas adicionais, como suporte nutricional, psicológico e social. Portanto, a DRC exige que os indivíduos realizem as sessões de hemodiálise com a frequência e o tempo indicados, tomem os medicamentos e cumpram um plano alimentar e de controle de líquidos, relativamente complexo e difícil. Não obstante, percebe-se que uma proporção significativa dessa população tem dificuldades de adesão ao tratamento (Maldaner, Beuter, Brondani, Budó \& Pauletto, 2008).

Os resultados deste estudo, em relação ao tratamento representado como difícil, coadunam-se com as elucidações de Santos e Valadares (2011) quando referem que inicialmente as pessoas acometidas pela doença renal associam a mesma ao tratamento da hemodiálise. Nesse direcionamento, doença e tratamento emergem como um todo indissociável, associando doença ao tratamento (diálise) e que este é uma mera continuidade.

Especificamente em relação ao grupo feminino, foi evidenciado que as dificuldades do tratamento possivelmente poderiam estar associadas ao papel construído sócio e culturalmente da figura feminina, como principal provedora dos cuidados da família, desde as atividades do lar às mais específicas como a saúde dos maridos e filhos (Cardoso, Vieira, Ricci \& Mazza, 2012).

De acordo com a OMS (2016) e Guerra-Guerrero, Sanhueza-Alvarado e Cáceres-Espina (2012), as mulheres são mais sensíveis aos sintomas físicos e mentais durante o processo do tratamento hemodialítico e mais afetadas aos sintomas da depressão do que os homens.

No que se refere ao segundo estímulo indutor "depressão", não foram observadas evocações no plano fatorial, conforme registro do subprograma ANECAR do software Trideux-Mots. Para o último estímulo "eu mesmo", essas mulheres com sintomas depressivos se representaram como "calmas" e "tristes". Essas elucidações estão ancoradas na esfera comportamental e psicoafetiva, respectivamente.

Apesar de esse grupo não ter apresentado evocações suficientes para compor o plano fatorial referente ao estímulo "depressão", pôde-se perceber que os mesmos se autoconceituaram por meio de um dos sintomas mais comuns do estado depressivo que é o elemento tristeza (APA, 2014). Portanto, o conhecimento do senso comum confirma a tristeza como elemento figurativo e elucida a importância desse sentimento quanto à identificação e manejo do quadro depressivo.

Ainda no eixo F2, no lado inferior do gráfico, situa-se o campo semântico do universo consensual dos pacientes independente do sexo sobre a DRC, a qual foi objetivada em "rim paralisado", sendo ancorada no conhecimento biomédico. Nesse sentido, essa representação está respaldada no conhecimento científico, que de acordo com o modelo biológico uma das principais características da doença renal em seu estágio avançado é a perda da função dos rins (KDIGO, 2013). 
O estímulo indutor "depressão" foi visto como sinônimo de "agonia" e ancorado na vertente psicoafetiva. Dessa forma, a depressão foi percebida como uma doença que causa sofrimento psíquico e advém de um mal-estar generalizado. Quanto ao terceiro estímulo "eu mesmo", este grupo, se autodefiniu de modo ambíguo, objetivado como pessoas "normais" e "doentes", ancorados na esfera psicossocial. Estes apontamentos remetem que a pessoa com DRC se considera como um ser normal, uma vez que pode desempenhar inúmeras atividades, e doentes quando vivenciam o tratamento e vê o corpo marcado pelas cicatrizes provocadas pelas fistulas, cateteres, exames e cirurgias. De acordo com Quintana e Müller (2006) por mais que o paciente negue a doença, essas marcas inseridas no corpo representam uma ruptura em suas vidas.

\section{Conclusão}

Com o objetivo principal de apreender as representações sociais acerca da Doença Renal Crônica e da depressão, elaboradas por pacientes sob tratamento de hemodiálise com e sem sintomas depressivos, pôde-se observar que a DRC foi ancorada, principalmente, na categoria biopsicossocial e no conhecimento biomédico. Apesar de a doença ser caracterizada pela paralisação dos rins, os participantes em geral afirmaram não ter noções da causa, percebendo a mesma no momento do diagnóstico e início do tratamento o qual foi representado como difícil. A depressão, por sua vez, foi representada como sinônimo de choro, falta de vontade para realizar as atividades da vida diária e agonia. Destaca-se que tais representações se encontraram relacionadas com as adversidades psicossociais vivenciadas no contexto da doença renal por este grupo de pertença.

Nesse sentido, estudar fenômenos como a doença renal e a depressão por meio da teoria das representações sociais possibilitou uma visão para além da cientificidade desses construtos, permitindo observar o conhecimento instintivo dos indivíduos, formulado a partir do convívio social, o qual é determinante do pensamento coletivo e das condutas frente a esses fenômenos, tanto no seu grupo de inserção como na sociedade em geral. Assim, o reconhecimento da importância do conhecimento espontâneo leva a um novo contorno de compreensão dessas doenças, de modo a transcender perspectivas medicalizantes, tornando possível o entrelaçamento de significados do ser humano enquanto ser multifacetado, permeado de aspectos sociais, biológicos, afetivos e psicológicos.

Diante do exposto, o fato de os pacientes desconhecerem as causas que desencadeiam a doença renal suscita a necessidade de maiores esclarecimentos à população como um todo, uma vez que algumas dessas causas poderiam ser prevenidas, o que contribuiria para o retardamento da emersão dos sintomas dessa doença. Salienta-se que se fazem necessárias intervenções dos profissionais que atuam diretamente com os pacientes renais, acerca da diferenciação entre doença e tratamento, especialmente, a hemodiálise, bem como mais explicações sobre esse procedimento, o que poderia contribuir para um melhor contorno emocional nos pacientes. Dessa forma, os resultados evidenciados neste artigo visaram contribuir para elaboração de estratégias de prevenção e tratamento da doença renal, que levem em consideração não apenas os aspectos físicos, mas, sobretudo, os psicossociais.

Como é peculiar a qualquer investigação científica, são evidenciadas algumas limitações. Recomenda-se para estudos futuros, a utilização de amostras representativas da população investigada, bem como equiparidade significativa no quantitativo dos subgrupos empregados na comparação com e sem sintomas depressivos. Destaca-se, também, que a 
prevalência da depressão encontrada não se trata de um diagnóstico, uma vez que foi utilizado apenas um instrumento para o rastreamento da depressão.

\section{Referências}

American Psychiatric Association - APA (2014). Manual Diagnóstico e Estatístico de Transtornos Mentais. 5 ed. (DSM-V). Porto Alegre: Artmed.

Andrade, S. V., Sesso, R., \& Diniz, D. H. M. P. (2015). Desesperança, ideação suicida e depressão em pacientes renais crônicos em tratamento por hemodiálise ou transplante. Jornal Brasileiro de Nefrologia, 37(1), 5563.

Botega, N. J., Bio, M. R., Zomignani, M. A., Garcia Jr, C., \& Pereira, W. A. B. (1995). Transtornos do humor em enfermaria de clínica médica e validação de escala de medida (HAD) de ansiedade e depressão. Revista Saúde Pública, 29(5), 359-363.

Campos, C. J. G., \& Turato, E. R. (2010). Tratamento Hemodialítico sob a ótica do doente renal: estudo clínico qualitativo. Revista Brasileira de Enfermagem, 63(5), 799-805.

Cardoso, L., Vieira, M. V., Ricci, M. A. M., \& Mazza, R. S. (2012). Perspectivas atuais sobre a sobrecarga do cuidador em saúde mental. Revista da Escola de Enfermagem da Universidade de São Paulo, 46(2), 513517.

Chilcot, J., Wellsted, D., Silva-Gane, M., \& Farrington, K. (2008). Depression on Dialysis. Nephron Clinical Practice, 108(4), 256 - 264.

Costa, F. G., \& Coutinho, M. P. L. (2016). Síndrome depressiva: um estudo com pacientes e familiares no contexto da doença renal crônica. Estudos Interdisciplinares em Psicologia, 7(1), 38-55.

Costa, F. G., Coutinho, M. P. L., Melo, J. R. F., \& Oliveira, M. X. (2014). Rastreamento da depressão no contexto da insuficiência renal crônica. Revista Temas em Psicologia, 22(2), 445-455.

Costa, M. S., Silva, M. J., Alves, M. D. S., Silva, W. P. P., \& Leite, M. K. L. (2007). Representaciones sociales de ancianos en hemodiálisis: un estudio de caso en el nordeste de Brasil. Revista Cubana de Enfermagem, 23(4), 1-7.

Coutinho, M. P. L. (2005). Depressão infantil e representações sociais (2ª ed). João Pessoa: Editora Universitária/ UFPB.

Coutinho, M. P. L., \& Costa, F. G. (2015). Depressão e insuficiência renal crônica: uma análise psicossociológica. Psicologia \& Sociedade, 27(2), 448449.

Cukor, D., Coplan, J, Brown, C., Peterson, R. A., \& Kimmel, P. L. (2008). Course of Depression and Anxiety Diagnosis in Patients Treated with Hemodialysis: A 16-month Follow-up. Clinical Journal of American Society of Nephrology, 3(6), 1752-1758.

Ferreira, P. L., \& Anes, E. J. (2010). Medição da qualidade de vida de insuficientes renais crônicos: criação da versão portuguesa do KDQOL-SF. Revista Portuguesa de Saúde Pública, 28(1), 31-39.

Garcia, T., Veiga, J. P. R., \& Motta, L. O. C. (2010). Comportamento depressivo e má qualidade de vida em homens com insuficiência renal crônica, submetidos à hemodiálise. Revista Brasileira de Psiquiatria, 32(4), 369-374.

Guerra-Guerrero, V., Sanhueza-Alvarado, O., \& Cáceres-Espina, M. (2012). Qualidade de vida de pessoas em hemodiálise crônica: relação com variáveis sociodemográficas, médico-clínicas e de laboratório. Revista Latino-Americana de Enfermagem, 20(5), 838-846.

Kaplan, H., Sadock, B. J., \& Grebb, J. A. (2007). Compêndio de psiquiatria: ciências do comportamento e psiquiatria clínica. Tradução: Dayse Batista. (9ạ ed.). Porto Alegre: Artmed.

Kidney Disease Improving Global Outcomes (KDIGO) (2013). KDIGO 2012 Clinical Practice Guideline for the Evaluation and Management of Chronic Kidney Disease. Kidney International Supplements, 3(1), 19-62.

Kimmel, P. L., Cohen, S. D., \& Peterson, R. A. (2008). Depression in patients with chronic renal disease: Where 
are we going? Journal of Renal Nutrition, 18(1), 99-103.

Maldaner, C. R., Beuter, M., Brondani, C. M., Budó, M. L. D., \& Pauletto, M. R. (2008). Fatores que influenciam a adesão ao tratamento na doença crônica: o doente em terapia hemodialítica. Revista Gaúcha de Enfermagem, 29(4), 647-653.

Marcolino, J. A. M, Mathias, L. A. S. T, Piccinini Filho, L., Guaratini, A. A., Suzuki, F. M., \& Alli, L. A. C. (2007). Escala Hospitalar de Ansiedade e Depressão: Estudo da Validade de Critério e da Confiabilidade com Pacientes no Pré-Operatório. Revista Brasileira de Anestesiologia, 57(1), 52-62.

Moscovici, S. (2012). Representações sociais: Investigações em psicologia social (7ạ ed.). (P. A. Guareschi, Trad.). Petrópolis, RJ: Vozes.

Nascimento, M. E. B., Campos, C. G. P., Mantovani, M. F., \& Cassi, C. C. (2015). Representações sociais sobre o adoecimento de pessoas com doença renal crônica. Revista Gaúcha de Enfermagem, 36(2), 106-112.

Nifa, S., \& Rudnicki, T. (2010). Depressão em pacientes renais crônicos em tratamento de hemodiálise. Revista da Sociedade Brasileira de Psicologia Hospitalar, 13(1), 64-75.

Nóbrega, S. M., \& Coutinho, M. P. L. (2011). A técnica de associação livre de palavras. In M. P. L. Coutinho \& E. R. A. Saraiva (Org). Métodos de pesquisa em psicologia social: perspectivas qualitativas e quantitativas. (pp. 95-106). Editora Universitária, João Pessoa/ UFPB.

Oliveira, S. G., \& Marques, I. R. (2011). Sentimentos do paciente portador de doença renal crônica sobre a autoimagem. Revista de Enfermagem da Universidade de Santo Amaro, 12(1), 38-42.

OMS (2016). World Health Organization. Depression. Recuperado de http://www.who.int/mediacentre/factsheets/fs369/en/.

Palmeira, A. T. (2009). Representações sociais de doença crônica: um estudo qualitativo com pessoas com diagnóstico de insuficiência renal ou dor crônica. Dissertação de Mestrado, Programa de Pós-Graduação em Psicologia, Universidade Federal da Bahia, Salvador, BA, Brasil.

Quintana, A. M., \& Muller, A. C. (2006). Da saúde à doença: representações sociais sobre a insuficiência renal crônica e o transplante renal. Revista Psicologia Argumento, 24(44), 73-80.

Ramos, I. C., Queiroz, M. V. O., \& Jorge, M. S. B. (2008). Cuidado em situação de Doença Renal Crônica: representações sociais elaboradas por adolescentes. Revista Brasileira de Enfermagem, 61(2), 193-200.

Ribeiro, K. C., Coutinho, M. P. L., \& Nascimento, E. S. (2010). Representação social da depressão em uma Instituição de Ensino da Rede Pública. Psicologia Ciência e Profissão, 30(3), 448-63.

Ribeiro, R. C. H. M., Oliveira, G. A. S. A., Ribeiro, D. F., Bertolin, D. C., Cesarino, C. B., Lima, L. C. E. Q., \& Oliveira, S. M. (2008). Caracterização e etiologia da insuficiência renal crônica em unidade de nefrologia do interior do Estado de São Paulo. Acta Paulista de Enfermagem, 21(Especial-Nefrologia), 207-211.

Rudnicki, T. (2014). Doença renal crônica: vivência do paciente em tratamento de hemodiálise. Contextos Clínicos, 7(1), 105-116.

Santos, A. F., Barbosa, R. B., Faro, S. R. S., \& Alves Júnior, A. (2005). Representações sociais do processo saúdedoença entre nefrologistas e pacientes renais crônicos. Psicologia, Saúde \& Doenças, 6, 57-67.

Santos, F. K., \& Valadares, G. V. (2011). Vivendo entre o pesadelo e o despertar: o primeiro momento no enfrentamento da diálise peritoneal. Escola Anna Nery, 15(1), 39-46.

Saraiva, E. R. A., \& Coutinho, M. P. L. (2008). O sofrimento psíquico no puerpério: um estudo psicossociológico. Revista Mal-estar e Subjetividade, 8(2), 505-27.

Smith, M. D., Hong, B. A., \& Robson, A. M. (1985). Diagnosis of depression in patients with end stage renal disease. The American Journal of Medicine, 79(2), 160-166.

Spinola, T. D., \& Gonçalves, V. M. S. (2012). Percepção de pacientes com insuficiência renal crônica quanto à interferência da fístula arteriovenosa em seu cotidiano. Revista Enfermagem Integrada, 5(2), 977-986. 